\title{
Forecasting New Cases of Bipolar Disorder Using Poisson Hidden Markov Model
}

\author{
Behnaz Alafchi', Saeid Yazdi-Ravandi ${ }^{2,3}$, Roya Najafi-Vosough' ${ }^{1}$, Ali Ghaleiha ${ }^{2}$, Majid Sadeghifar ${ }^{4 *}$ \\ ${ }^{1}$ Department of Biostatistics, School of Public Health, Hamadan University of Medical Sciences, Hamadan, Iran. \\ ${ }^{2}$ Behavioral Disorders and Substance Abuse Research Center, Hamadan University of Medical Sciences, Hamadan, Iran \\ ${ }^{3}$ Young Researchers and Elite Club, Rudehen Branch, Islamic Azad University, Rudehen, Iran \\ ${ }^{4}$ Department of Statistics, Faculty of Basic Sciences, Bu-Ali Sina University, Hamadan, Iran
}

\begin{abstract}
Background: Bipolar disorder (BD) is a major public health problem. In time series count data there may be over dispersion, and serial dependency. In such situation some models that can consider the dependency are needed. The purpose current research was to use Poisson hidden Markov model to forecast new monthly BD instances.

Methods: In current study the dataset including the frequency of new instances of BD from October 2008 to March 2015 in Hamadan Province, the west of Iran were used. We used Poisson hidden Markov with different number of conditions to determine the best model according to Akaike Information Criterion (AIC) and Bayesian Information Criterion (BIC). Then we used final model to forecast for the next 24 months.

Results: Poisson hidden Markov with two states were chosen as the final model. Each component of dependent mixture model explained one of the states. The results showed that the new BD cases is increase over time and due to forecasting results number of patients for the next 24 months comforted in state two with mean 85.15 . The forecast interval was approximately $(56,100)$.

Conclusion: As the Poisson hidden Markov models was not used to forecast the future states in other prior researches, the findings of this study set forward a forecasting strategy as an alternative to common methods, by considering its deficiencies.

Keywords: Bipolar Disorder; Forecast; Poisson hidden Markov model; Hamadan
\end{abstract}

*Correspondence to Majid Sadeghifar, PhD; Department of statistics, Faculty of Basic Science, Bu-Ali Sina University, Hamadan, Iran.

Tel: +980918 8115417 Fax: +9808138271541 Email: sadeghifar@basu.ac.ir

Published online March 15, 2018

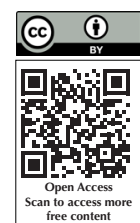

\section{Introduction}

Bipolar disorder (BD) is common disorder and a major public health problem. This disorder is severing chronic and accompanied by problems in the person's psychosocial functioning. ${ }^{1}$ Based on the DSM-IV-TR, a diagnosis of (BD) requires manic, hypomanic, or mixed episodes. ${ }^{2}$ Adding to the suffering of this patients and their families, the condition significantly increases costs for society. ${ }^{3}$

There is BD in all over the world. Lifetime prevalence rates of bipolar I disorder are estimated to range from 1.6 to $0.2 \%{ }^{4}$ Over $90 \%$ of patients with BD experience recurrences during their lifetimes. ${ }^{5}$ The relapse rate in the over periods of 2 years is close to $60 \%$, and over 5 years, $75 \% .{ }^{6,7}$ According to the reported of WHO, BD is the 6 worldwide prominent reason of life disability worldwide among persons aged 15 to 44 years. ${ }^{8}$

To identifying such risk factors it can be used to compare incident cases to non-cases. The annual incidence of bipolar illness is less than 1 percent. As the incidence of $\mathrm{BD}$ is low, it is difficult to estimate, because milder forms of $\mathrm{BD}$ are often missed. Most incidence researches in $\mathrm{BD}$ identify cases at admission. ${ }^{1,9-11}$

We used statistical methods for the analysis of time series $\mathrm{BD}$. When the observations are a sequence of unbounded counts, using regular time series models that are established on continuous outcomes with continuous distribution are not suitable. ${ }^{11,12}$ The Poisson distribution is a natural choice to describe such data. The most important exclusivity of this distribution is the equality of the mean and the variance. But often this exclusivity will not hold and variance is bigger than the mean, which is called over dispersion. Using mixture models is one way to handle this problem. ${ }^{12}$ As when the data are a series of unbounded counts there may be over dispersion, and serial dependency, using independent Poisson random variables are also not appropriate and some models that incorporate this dependency are needed. ${ }^{12}$

Hidden Markov model can accommodate both over dispersion and serial dependency by allowing the probability distribution of each observation to depend on

(C) 2018 The Author(s). This is an open access article distributed under the terms of the Creative Commons Attribution License (http:// creativecommons.org/licenses/by/4.0/), which permits unrestricted use, distribution, and reproduction in any medium, provided the original work is properly cited. 
the unobserved state of a Markov chain. ${ }^{12}$ The hidden part of this model use Markov property that means, occupied state at each step depends only on the previous state. ${ }^{14}$ Although hidden Markov model has been used in different areas, it is not common in medical and psychological sciences. Due to the importance of knowing frequency of $\mathrm{BD}$ patients for the preventive action, we need number of these patients for next times.

In the current research, we aimed to use Poisson hidden Markov (PHM) model as the forecast method to predict the number of patients that diagnosis whit BD.

\section{Materials and Methods}

\section{Data Collection}

In the present dataset including the frequency of new instances of BD from October 2008 to March 2015 in Hamadan Province, the west of Iran was used. This data belongs to the patients with new recurrence leading to hospitalization in the Farshchian Sina hospital.

\section{Hidden Markov Model}

Hidden Markov model is a special kind of mixture model. This model consists of two parts:

The first part is hidden states $\left\{C_{t}\right\}$, which have Markov property and is called parameter processes, and the second one is an observed parameter $\left\{X_{t}\right\}$, state-dependent process. $X_{t}$ depends on the present state of $C_{t}$ and not on prior states. The observation of this part are independent conditionally on hidden states. The model is represented as follows:

$$
\begin{aligned}
& P\left(C_{t} \mid C_{1}, C_{2}, \ldots, C_{t-1}\right)=P\left(C_{t} \mid C_{t-1}\right), \quad t=1,2, \ldots \\
& P\left(X_{t} \mid X_{1}, X_{2}, \ldots, X_{t-1}, C_{1}, C_{2}, \ldots, C_{t}\right)=P\left(X_{t} \mid C_{t}\right), \\
& t \in N .
\end{aligned}
$$

Let $C_{t}$ represent an irreducible Markov chain and has $\mathrm{m}$ states, then we call $X_{t}$ an $\mathrm{m}$-state hidden Markov model by the transition matrix $\Gamma=\left(\gamma_{i j}\right)$. Hidden Markov model involves both estimating the elements of the transition matrix $\gamma_{i j}=P\left(C_{t}=j \mid C_{t-1}=i\right)$ and the parameter of model $\lambda_{i}$ to estimate $\mathrm{P}_{\mathrm{i}}(\mathrm{x})$. For discrete observations, $\mathrm{P}_{\mathrm{i}}(\mathrm{x})$ demonstrate possibility mass function of $X_{-} t$ at time $t$ if the Markov chainis in statei. For Poisson observation model it define as,

$$
P_{i}(x)=P\left(X_{t}=x \mid C_{t}=i\right)=\frac{e^{\lambda_{i}} \lambda_{i}^{x}}{x !}
$$

Parameters are obtained by maximum likelihood estimation. The likelihood function of the observed data $L_{T}$ can be shown by:

$$
L_{T}=\delta P\left(x_{1}\right) \Gamma P\left(x_{2}\right) \ldots \Gamma P\left(x_{2}\right) 1^{\prime}
$$

Where $\delta$ the distribution of is $\mathrm{C}_{1}, \mathrm{P}(\mathrm{X})$ is diagonal matrix with the element $\mathrm{P}_{\mathrm{i}}(\mathrm{x})$.

Making predictions about the future given the history of the process is the other purpose of the study. Forecasting distribution can be acquired from this equation:

$P\left(X_{T+h}=x \mid X^{(T)}=x^{(T)}\right)=\frac{\alpha_{T} \Gamma^{h} \mathrm{P}(\mathrm{x}) 1^{\prime}}{L_{T}}$

Where

$$
\alpha_{t}=\delta P\left(x_{1}\right) \Gamma P\left(x_{2}\right) \ldots \Gamma P\left(x_{t}\right) \quad t=1,2, \ldots, T
$$

And $\alpha_{T}=\alpha_{T-1} \Gamma P\left(x_{t}\right)$.

At first step, we examine different stationary PHM models to specify the proper number of the state. To compare the models with different status, Akaike Information Criterion (AIC) and Bayesian Information Criterion (BIC) are used. For both criterions, the best model is the one that minimizes the information criterion. Then the number of states in the best model consider as the dimension of transition. The transition probability matrix consists of the probability of transition from one state to the other and the probability of staying in each state. The parameters are estimated in the maximum likelihood framework. ${ }^{14}$

Forecasting was calculated for the next 12 months. And the data for the first 6 months mean April to September 2015 used to check the model prediction ability. In this study available codes provided by Zucchini and MacDonald are used and all calculations were carried out by R.3.2.2 software.

\section{Results}

Data included the series of monthly counts of new BD cases for 79 months that contains 6336 patients. The mean and variance of new bipolar instances monthly were 80.20 and 173.11, respectively, which shows strong over dispersion in accordance with Poisson distribution. Figure 1 shows the trend of data. The maximum and minimum numbers were 45 and 114 that showed the wide range of observations.

The results of fitting PHM models with different number of states showed model with two states has the least AIC and BIC of all (Table 1).

Maximum likelihood method was used to estimate of parameters for transition matrix, probability of staying in each state and the parameter for Poisson distribution. The likelihood of direct transition from state 1 to state 2 was 0.24 and the opposite was 0.07 . The probabilities of staying in state 1 and state 2 are 0.76 and 0.93 respectively. These indicate a more persistent process that tends to stay in a certain state more than transition to the other, specifically when the chain is in state 2 . It means that it is expected to stay in a given state for a long time. Table 2 showed transition matrix. The mean number of new cases 


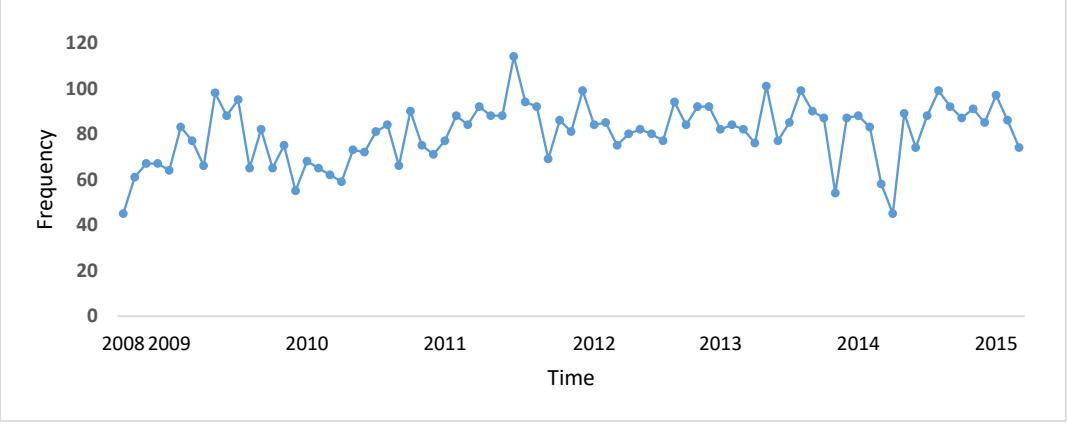

Figure 1. Frequency of New Cases With Bipolar disorder from October 2008 to March 2015.

in two states were 62.41 and 85.15. Min and Maximum of new BD instances in cluster related to state 1 and 2 were $(45,75)$ and $(76,114)$.

The results of prediction state for 12 next months showed that the frequency of new cases for all these months with a probability greater than 0.77 lies in state 2 by mean of 62.41 . Details of forecasting results are represented in Table 3. Monthly forecast mean varies from 79.74 to 82.47 numbers in month with the intervals (56, $99)$ and $(61,100)$. The data for all the first 6 months were in the range of observation in state 2 that are consistent with model predictions.

\section{Discussion}

The findings of the present study illustrated that the new $\mathrm{BD}$ instances were increased over time, as all predicting counts lying in state 2 with mean 85.15. The available data for the first 6 months confirmed the predicting results. Although the available data was limited and more data may be needed, it can be concluded that the model has an acceptable predicting power. According to interval forecasts the new $\mathrm{BD}$ cases can vary approximately from 56 to 100 . It should be considered that these estimates are reliable if other factors have been constant during the forecasting time.

Table 1. Comparison of Stationary Poisson Hidden Markov Models With Different States

\begin{tabular}{lll}
\hline Model & AIC & BIC \\
\hline 1-state PHM & 668.00 & 670.37 \\
2-state PHM & 619.48 & 628.95 \\
3-state PHM & 629.47 & 650.80 \\
\hline
\end{tabular}

Abbreviations: PHM, Poisson Hidden Markov; AIC, Akaike Information Criterion; BIC, Bayesian Information Criterion.

Table 2. Estimation of Transition Probability

\begin{tabular}{lcc}
\hline & State 1 & State 2 \\
\hline State 1 & 0.76 & 0.24 \\
State 2 & 0.07 & 0.93 \\
\hline
\end{tabular}

Hidden Markov model are used to model a series that are believed to transition over a finite set of unobserved states. However, we found a limited number of studies that applied this model to forecasting next states. Some studies showed that admission rates of bipolar patients increased in some months of year such as during spring and summer. But they used usual time series model like ARIMA to determining the status (high/low bipolar cases in each month). Their findings revealed a mixture of two dynamics with a low and higher level. ${ }^{15-17}$ As we noted, hidden Markov model is a kind of mixture model that in which forecasting the next status is possible. This model also has some benefits: overall capability and flexibility to manage missing data. Many researchers have been used hidden Markov models in different areas for various purposes.

Adriana Lopez used mixture of first order Markov chains and Hidden Markov models for longitudinal course of youth BD. The findings of their study illustrated that the combination of four first order Markov chains found patterns of movers and stayers and hidden Markov model with ten hidden states. He considered sex, age of bipolar onset and socio-economic status as covariates in extended hidden Markov models. ${ }^{18}$

DeSantis and Bandyopadhyay used Hidden Markov models for zero inflated Poisson counts. They found that zero-inflated PHM model outperforms other models for longitudinal count up data. ${ }^{19}$ Hamaker et al used the threshold autoregressive model and the Markov turning autoregressive model to model the psychological processes that are characterized by recurrent shifts between different conditions. Their research was at the level of the individual. They applied these models to daily data on manic and depressive condition for individuals with rapid-cycling BD. ${ }^{20}$ Cooper and Lipsitch analyzed hospital infections data by using hidden Markov models. They suggested the structured hidden Markov model that is depended on a mechanistic model of the epidemic process is more biologically acceptable, and allows key epidemiological variable to be estimated. ${ }^{21}$

The findings of this study need to be interpreted within 
Table 3. Result of Forecasting State for 2 State Poisson Hidden Markov

\begin{tabular}{|c|c|c|c|c|c|c|c|c|c|c|c|c|}
\hline Month & 1 & 2 & 3 & 4 & 5 & 6 & 7 & 8 & 9 & 10 & 11 & 12 \\
\hline Forecast mode & 85 & 85 & 84 & 84 & 84 & 84 & 84 & 84 & 84 & 84 & 84 & 84 \\
\hline Forecast median & 84 & 83 & 83 & 82 & 82 & 82 & 82 & 82 & 82 & 82 & 82 & 82 \\
\hline Forecast mean & 82.47 & 81.62 & 81.03 & 8.63 & 80.35 & 80.16 & 80.03 & 79.94 & 79.88 & 79.84 & 79.81 & 79.79 \\
\hline $\begin{array}{l}\text { Nominal forecast } \\
90 \% \text { interval }\end{array}$ & {$[61,100]$} & {$[59,100]$} & {$[58,100]$} & {$[57,100]$} & {$[57,99]$} & {$[57,99]$} & {$[56,99]$} & {$[56,99]$} & {$[56,99]$} & {$[56,99]$} & {$[56,99]$} & {$[56,99]$} \\
\hline $\begin{array}{l}\text { Probability of } \\
\text { State } 1\end{array}$ & 0.11 & 0.15 & 0.18 & 0.20 & 0.21 & 0.22 & 0.22 & 0.22 & 0.23 & 0.23 & 0.23 & 0.23 \\
\hline $\begin{array}{l}\text { Probability of } \\
\text { State } 2\end{array}$ & 0.89 & 0.58 & 0.82 & 0.80 & 0.79 & 0.78 & 0.78 & 0.78 & 0.77 & 0.77 & 0.77 & 0.77 \\
\hline
\end{tabular}

the context of two limitations. First of all, we just focused on the frequency of new BD cases that their disease was severe and hospitalized. Furthermore, many bipolar patients may not be admitted to hospitals. The other one is that we considered the frequency of new BD cases subtypes of mood episodes. After all our suggestion for next study is to apply the PHM models in the presence of covariates such as age at diagnosis, sex, mood episode, and etc.

\section{Conclusion}

As the PHM models was not used to forecast the future states in other prior researches, the findings of this study set forward a forecasting strategy as an alternative to common methods, by considering its deficiencies.

\section{Conflict of Interest Disclosures}

The authors declare that they have no conflict of interests.

Ethical Statement

Not applicable.

\section{Acknowledgement}

We would like to thank the staff of the Farshchian-Sina hospital for their collaboration.

\section{References}

1. Sadock BJ, Sadock VA. Kaplan and Sadock's Synopsis of Psychiatry: Behavioral Sciences/Clinical Psychiatry. Lippincott Williams \& Wilkins; 2011

2. American Psychiatric Association. Diagnostic and Statistical Manual of Mental Disorders. 4th ed. Washington, DC: American Psychiatric Association; 2000.

3. Brunello N, Burrows GD, Jonsson B, Judd LL, Kasper S, Keller MB, et al. Critical issues in the treatment of affective disorders. Depression. 1995;3(4):187-98. doi: doi:10.1002/ depr.3050030406.

4. Angst J, Marneros A. Bipolarity from ancient to modern times: conception, birth and rebirth. J Affect Disord. 2001;67(1-3):319.

5. Solomon DA, Keitner GI, Miller IW, Shea MT, Keller MB. Course of illness and maintenance treatments for patients with bipolar disorder. J Clin Psychiatry. 1995;56(1):5-13.

6. Gelenberg AJ, Kane JM, Keller MB, Lavori P, Rosenbaum JF, Cole K, et al. Comparison of standard and low serum levels of lithium for maintenance treatment of bipolar disorder. N Engl J Med. 1989;321(22):1489-93. doi: 10.1056/ nejm198911303212201.

7. Gitlin MJ, Swendsen J, Heller TL, Hammen C. Relapse and impairment in bipolar disorder. Am J Psychiatry.
1995;152(11):1635-40. doi: 10.1176/ajp.152.11.1635.

8. Murray CJ, Lopez AD. The global burden of disease and injury series, volume 1: a comprehensive assessment of mortality and disability from diseases, injuries, and risk factors in 1990 and projected to 2020. Geneva : World Health Organization; 1996.

9. Kennedy N, Everitt B, Boydell J, Van Os J, Jones PB, Murray RM. Incidence and distribution of first-episode mania by age: results from a 35-year study. Psychol Med. 2005;35(6):85563.

10. Laursen TM, Munk-Olsen T, Nordentoft M, Bo Mortensen P. A comparison of selected risk factors for unipolar depressive disorder, bipolar affective disorder, schizoaffective disorder, and schizophrenia from a danish population-based cohort. J Clin Psychiatry. 2007;68(11):1673-81.

11. Lloyd T, Kennedy N, Fearon P, Kirkbride J, Mallett R, Leff J, et al. Incidence of bipolar affective disorder in three UK cities: results from the AESOP study. Br J Psychiatry. 2005;186:12631. doi: 10.1192/bjp.186.2.126.

12. Zucchini W, MacDonald IL. Hidden Markov models for time series: an introduction using R. CRC Press; 2009.

13. Ghaffari ME, Ghaleiha A, Taslimi Z, Sarvi F, Amini P, Sadeghifar $M$, et al. Forecasting Schizophrenia Incidence Frequencies Using Time Series ApproachInt Clin Neurosci J. 2017;4(4):152-6. doi: 10.15171/icnj.2017.06.

14. Inge A. Hidden Markov Models. Sweden: Stockholm University; 2013.

15. Lee HC, Tsai SY, Lin HC. Seasonal variations in bipolar disorder admissions and the association with climate: a population-based study. J Affect Disord. 2007;97(1-3):61-9. doi: 10.1016/j.jad.2006.06.026.

16. Shapira A, Shiloh R, Potchter O, Hermesh H, Popper M, Weizman A. Admission rates of bipolar depressed patients increase during spring/summer and correlate with maximal environmental temperature. Bipolar Disord. 2004;6(1):90-3.

17. Bonsall MB, Wallace-Hadrill SM, Geddes JR, Goodwin GM, Holmes EA. Nonlinear time-series approaches in characterizing mood stability and mood instability in bipolar disorder. Proc Biol Sci. 2012;279(1730):916-24. doi: 10.1098/ rspb.2011.1246.

18. Lopez A. Markov models for longitudinal course of youth bipolar disorder. ProQuest; 2008.

19. DeSantis SM, Bandyopadhyay D. Hidden Markov models for zero-inflated Poisson counts with an application to substance use. Stat Med. 2011;30(14):1678-94. doi: 10.1002/sim.4207.

20. Hamaker EL, Grasman RP, Kamphuis JH. Regime-switching models to study psychological processes. In: Molenaar PCM, Newell KM. Individual pathways of change: Statistical models for analyzing learning and development. Washington, DC, US: American Psychological Association; 2010:155-168. doi: 10.1037/12140-009.

21. Cooper B, Lipsitch M. The analysis of hospital infection data using hidden Markov models. Biostatistics. 2004;5(2):223-37. doi: 10.1093/biostatistics/5.2.223. 